\title{
A NOTE ON THE COSEGRE CLASS OF A SUBVARIETY*
}

\author{
SŌICHI KAWAI ${ }^{\dagger}$
}

In this short note we introduce the notion of the cosegre class of a subvariety of a nonsigular algebraic variety, which is more geometric than the Segre class of a subvariety, and with it give a proof, which is essentially in the framework of algebraic geometry, to a theorem of Brylinski,Dubson and Kashiwara[1,Corollary 5].

Let $Z$ be a subvariety of an algebraic variety $X$ and denote by $C_{Z} X$ the normal cone to $Z$ in $X$. In Fulton[2] the Segre class $\mathrm{s}(Z, X)$ of $Z$ in $X$ is defined to be the Segre class of $C_{Z} X$. The normal cone $C_{Z} X$ is defined to be $\operatorname{Spec}\left(\sum \mathcal{I}^{k} / \mathcal{I}^{k+1}\right)$, where $\mathcal{I}$ is the ideal sheaf defining $Z$ in $X$ and has the projective completion $q: P\left(C_{Z} X \oplus 1\right) \rightarrow Z$ with the canonical line bundle $\mathcal{O}(1)$. The Segre class $\mathrm{s}(Z, X)$ is defined as

$$
\mathrm{s}(Z, X)=q_{*}\left(\sum_{i} c_{1}(\mathcal{O}(1))^{i} \cap\left[P\left(C_{Z} X \oplus 1\right)\right]\right) .
$$

Hereafter we assume that $X$ is a nonsingular algebraic variety of dimension $n$ and $Z$ is an irreducible subvariety of $X$. For simlplicity's sake, we assume that $\operatorname{dim} Z<\operatorname{dim} X$. In this note cycles are always algebraic cycles and the intersection of cycles are the refined intersection of Fulton[2] in Borel-Moore homology groups. Let $T_{Z_{s p}}^{*} X$ be the conormal bundle to the nonsingular part $Z_{s p}$ of $Z$ in $X$. Then the closure of $T_{Z_{s p}}^{*} X$ in the cotangent bundle $T^{*} X$ is a conic subvariety, which we denote simply by $T_{Z}^{*} X$. The closure of $T_{Z}^{*} X$ in the projective completion $P\left(T^{*} X \oplus 1\right)$ is denoted by $\frac{Z}{T_{Z}^{*} X}$. Let $q: P\left(T^{*} X \oplus 1\right) \rightarrow X$ be the projection and $\mathcal{O}(1)$ the canonical line bundle on $P\left(T^{*} X \oplus 1\right)$. We call

$$
s^{*}(Z, X)=q_{*}\left(\sum_{i} c_{1}(\mathcal{O}(1))^{i} \cap\left[\overline{T_{Z}^{*} X}\right]\right)
$$

the cosegre class of $Z$ in $X$. We define linearly the cosegre class $s^{*}(z, X)$ of a cycle $z$ on $X$, which is an element of $H .(|z|, \mathbb{Z})$, where $|z|$ is the support of $z$ and $\mathbb{Z}$ is the ring of integers. The following lemma is obtained in Sabbah[7,Lemma 1.2.1].

Lemma. Let $\check{c}_{M}(Z)$ be the checked Mather-Chern class of $Z$ and $c\left(T^{*} X\right)$ the Chern class of the cotangent bundle of $X$. Then we have

$$
\check{c}_{M}(Z)=c\left(T^{*} X\right) \cap s^{*}(Z, X) .
$$

Letting $\mathcal{M}$ be a bounded complex of $\mathcal{D}_{X}$ modules with regular holonomic cohomology. Letting $\operatorname{Ch}\left(\mathcal{M}^{\cdot}\right)=\sum m_{j} T_{V_{j}}^{*} X$ be the characteristic cycle of $\mathcal{M}$; where $\left\{V_{j}\right\}$ is a stratification of $X$, we define the checked Mather-Chern class $\check{c}_{M}\left(\mathcal{M}^{*}\right)$ to be

$$
\check{c}_{M}\left(\mathcal{M}^{\cdot}\right)=\sum m_{j} \check{c}_{M}\left(\bar{V}_{j}\right)
$$

\footnotetext{
* Received December 21, 1999; accepted for publication July 19, 2000.

†Department of Mathematics, Rikkyo University, Tokyo 171-8501, Japan (kawai@rkmath. rikkyo.ac.jp).
} 
In this note we denote the cycle $\sum m_{j} \overline{T_{V_{j}}^{*} X}$ on $T^{*} X$ by the same letter $\operatorname{Ch}\left(\mathcal{M}^{*}\right)$. By the above lemma we have

$$
\check{c}_{M}\left(\mathcal{M}^{*}\right)=c\left(T^{*} X\right) \cap s^{*}\left(\sum m_{j} \bar{V}_{j}, X\right)=c\left(T^{*} X\right) \cap q_{*}\left(\sum c_{1}(\mathcal{O}(1))^{i} \cap \operatorname{Ch}\left(\mathcal{M}^{*}\right)\right) .
$$

Let $Y$ be a nonsingular algebraic variety of dimension $m$ and $f: X \rightarrow Y$ a projective map. Let $q_{Y}: f^{*} T^{*} Y \rightarrow X$ be the induced bundle, $\tilde{f}: f^{*} T^{*} Y \rightarrow T^{*} Y$ the projection and $\alpha_{Y}$ the canonical 1-form on $T^{*} Y$. Then $\tilde{f}^{*}\left(\alpha_{Y}\right)$ may be considered to be a section of the induced bundle $q_{Y}^{*} T^{*} X$ over $f^{*} T^{*} Y$.

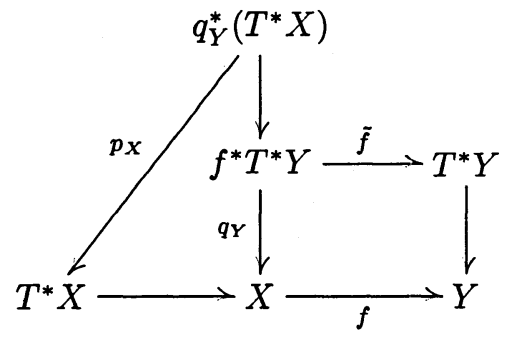

The following theorem is a corollary of a theorem of Ginsburg[3](cf. Kawai[5])

Theorem 1. Let $\int_{f} \mathcal{M} \cdot$ be the direct image of $\mathcal{M} \cdot$ by the map $f$. Then we have

$$
\operatorname{Ch}\left(\int_{f} \mathcal{M}^{*}\right)=\tilde{f}_{*}\left(p_{X}^{*}\left(\operatorname{Ch}\left(\mathcal{M}^{*}\right)\right) \cdot \tilde{f}^{*}\left(\alpha_{Y}\right)\left(\left[f^{*} T^{*} Y\right]\right)\right) .
$$

Here the intersection is the intersection in $q_{X}^{*}\left(T^{*} X\right)$ and $\tilde{f}^{*}\left(\alpha_{Y}\right)\left(\left[f^{*} T^{*} Y\right]\right)$ is the image of the the cycle $\left[f^{*} T^{*} Y\right]$ by the section $\tilde{f}^{*}\left(\alpha_{Y}\right)$. The intersection is considered to be a cycle in $f^{*} T^{*} Y$ which is identified with the image of the section.

As for the direct image of the cosegre class by the map $f$ we have the following theorem if we assume for simplicity's sake that $\operatorname{dim} X \leq \operatorname{dim} Y$.

Theorem 2.. Let $\bar{q}_{Y}: P\left(f^{*} T^{*} Y \oplus 1\right) \rightarrow X$ be the projective completion of the vector bundle $f^{*} T^{*} Y \rightarrow X, i: f^{*} T^{*} Y \rightarrow P\left(f^{*} T^{*} Y \oplus 1\right)$ the canonical injection and $\overline{p_{X}^{*}\left(T_{Z}^{*} X\right) \cdot \tilde{f}^{*}\left(\alpha_{Y}\right)\left(\left[f^{*} T^{*} Y\right]\right)}$ the cycle which is obtained as the closure of $p_{X}^{*}\left(T_{Z}^{*} X\right)$. $\tilde{f}^{*}\left(\alpha_{Y}\right)\left(\left[f^{*} T^{*} Y\right]\right)$ in $P\left(f^{*} T^{*} Y \oplus 1\right)$. Then we have

$$
\left.c\left(T^{*} X\right) \cap s^{*}(Z, X)=c\left(f^{*} T^{*} Y\right) \cap \bar{q}_{Y *}\left(s\left(L_{Y}\right) \cap \overline{\left[p_{X}^{*}\left(T_{Z}^{*} X\right) \cdot \tilde{f}^{*}\left(\alpha_{Y}\right)\left(\left[f^{*} T^{*} Y\right]\right)\right.}\right]\right),
$$

where $L_{Y}$ is the tautological line bundle on $P\left(f^{*} T^{*} Y \oplus 1\right)$.

We have the following corollary which is equivalent to Corollary 5 of Brylinski,Dubson and Kashiwara[1] and the theorem of MacPherson via the RiemannHilbert corresopondence.

Corollary. We have

$$
\check{c}_{M}\left(\int_{f} \mathcal{M}^{\cdot}\right)=f_{*}\left(\check{c}_{M}\left(\mathcal{M}^{\cdot}\right)\right)
$$

\section{Proof of Theorem 2 and Corollary}

For the proof of the theorem we prepare a lemma. 
Lemma Let $E, F$ be vector bundles of rank $n, m$ respectively on an algebraic variety $Z$. Let $\bar{p}: P(E \oplus F \oplus 1) \rightarrow Z(\bar{q}: P(F \oplus 1) \rightarrow Z$, resp. $)$ be the projective completion of the vector bundle $E \oplus F \oplus 1(F \oplus 1$, resp. $)$. We consider $P(F \oplus 1)$ and the projective bundle $P(E)$ to be the subvarieties of $P(E \oplus F \oplus 1)$. Let $\varpi: \tilde{P} \rightarrow P(E \oplus F \oplus 1)$ be the blow-up of $P(E \oplus F \oplus 1)$ along $P(E)$. Then the projection of $P(E \oplus F \oplus 1)$ to $P(F \oplus 1)$ with center $P(E)$ induces the morphism $\pi: \tilde{P} \rightarrow P(F \oplus 1)$. Let $L$ be the tautological line bundle on the projective bundle $P(F \oplus 1)$. Then $\pi: \tilde{P} \rightarrow P(F \oplus 1)$ is equivalent to the projective bundle $P\left(\bar{q}^{*} E \oplus L\right) \rightarrow P(F \oplus 1)$. Identifying them, we have

$$
\varpi^{*} \mathcal{O}_{P(E \oplus F \oplus 1)}(1)=\mathcal{O}_{P\left(\bar{q}^{*} E \oplus L\right)}(1) .
$$

We identify $P\left(\bar{q}^{*} E \oplus L\right)=P\left(\left(L^{-1} \otimes \bar{q}^{*} E\right) \oplus 1\right)$. Then the exceptional divisor $\Theta$ with respect to the blowing up is equal to the subvariety $P\left(\bar{q}^{*} E\right)=P\left(L^{-1} \otimes \bar{q}^{*} E\right)$ and the line bundle $\mathcal{O}_{\tilde{P}}(\Theta)$ associated to the divisor $\Theta$ is $\pi^{*}\left(L^{-1}\right) \otimes \mathcal{O}_{P\left(\bar{q}^{*} E \oplus L\right)}(1)$.

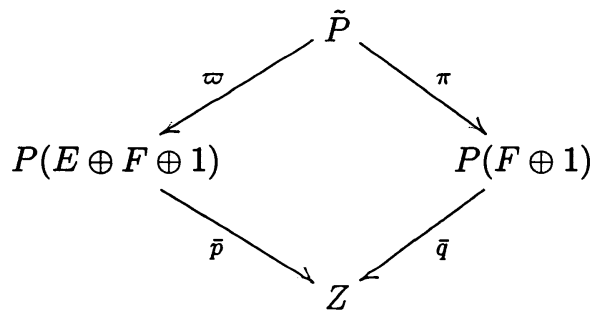

Proof. Let $\mathcal{O}_{P(E)}(1)$ be the canonical line bundle of $P(E)$. The normal bundle to $P(E)$ in $P(E \oplus F \oplus 1)$ is isomorphic to $\mathcal{O}_{P(E)}(1) \otimes(F \oplus 1)$. Hence we may consider $\tilde{P}$ to be the subvariety of $P(E \oplus F \oplus 1) \times{ }_{Z} P\left(\mathcal{O}_{P(E)}(1) \otimes(F \oplus 1)\right)=P(E \oplus F \oplus$ 1) $\times_{Z} P(F \oplus 1)$ and $\pi: \tilde{P} \rightarrow P(F \oplus 1)$ to be the projection $P(E \oplus F \oplus 1) \times{ }_{Z}$ $P(F \oplus 1) \rightarrow P(F \oplus 1)$. Let $U_{\alpha}$ be an open subset of $Z$ and $E \mid U_{\alpha}=U_{\alpha} \times \mathbb{C}^{n}$ with fiber coordinates $\left(x_{\alpha, 0}, \ldots, x_{\alpha, n-1}\right)\left(F \mid U_{\alpha}=U_{\alpha} \times \mathbb{C}^{m}\right.$ with fiber coordinates $\left(x_{\alpha, n}, \ldots, x_{\alpha, n+m-1}\right), 1 \mid U_{\alpha}=U_{\alpha} \times \mathbb{C}$ with fiber coordinates $x_{\alpha, n+m}$, respectively ) a local trivialization of the vector bundle $E$ ( $F, 1$, respectively). We consider a copy of vector bundle $F \oplus 1$ and a local trivialization $U_{\alpha} \times \mathbb{C}^{m+1}$ of the copy with fiber coordinates $\left(y_{\alpha, 0}, \ldots, y_{\alpha, m}\right)$. Then $\tilde{P}$ is considered to be the subvariety of $U_{\alpha} \times$ $P\left(\mathbb{C}^{n} \times \mathbb{C}^{m} \times \mathbb{C}\right) \times P\left(\mathbb{C}^{m+1}\right)$ defined by the equations

$$
x_{\alpha, n+i} y_{\alpha, j}=x_{\alpha, n+j} y_{\alpha, i}, \quad 0 \leq i, j \leq m .
$$

Let $V_{\alpha, j}$ be the subset of $P(E \oplus F \oplus 1) \mid U_{\alpha}$ such that $x_{\alpha, j} \neq 0$. A system of coordinate transformations $\left\{g_{(\beta, j),(\alpha, i)}\right\}$ of the canonical line bundle $\mathcal{O}_{P(E \oplus F \oplus 1)}(1)$ with respect to the covering $\left\{V_{\alpha, i}\right\}$ is given by $g_{(\beta, j),(\alpha, i)}=x_{\alpha, i} / x_{\beta, j}$ for $V_{\alpha, i} \cap V_{\beta, j} \neq \emptyset$. Let $W_{\alpha, i}$ be the open subset of $P(F \oplus 1) \mid U_{\alpha}$ such that $y_{\alpha, i} \neq 0$. Then the map $\psi_{\alpha, i}$ : $\pi^{-1}\left(W_{\alpha, i}\right) \rightarrow W_{\alpha, i} \times \mathbb{P}^{n}$, where $\mathbb{P}^{n}$ is a projective space with homogeneous coordinates $\left(\lambda_{\alpha, 0}, \ldots, \lambda_{\alpha, n}\right)$, defined to be

$$
\left(\lambda_{\alpha, 0}, \ldots, \lambda_{\alpha, n}\right)=\left(x_{\alpha, 0}, \ldots, x_{\alpha, n-1}, x_{\alpha, n+i}\right)
$$

is a well-defined morphism. In fact, if $x_{\alpha, n+i}=0$, by the above defining equations of $\tilde{P} \mid U_{\alpha}$ we have $x_{\alpha, n+j}=x_{\alpha, n+i} \frac{y_{\alpha, j}}{y_{\alpha, i}}$ and hence $x_{\alpha, n+j}=0$, for $j=0, \ldots, m$. Therefore we have $\left(x_{\alpha, 0}, \ldots, x_{\alpha, n-1}\right) \neq 0$. We infer readily that the morphism $\psi_{\alpha, i}$ 
is an isomorphism and $\pi: \tilde{P} \rightarrow P(F \oplus 1)$ is equivalent to the projective bundle $P\left(\bar{q}^{*} E \oplus L\right) \rightarrow P(F \oplus 1)$. We identify them. If $x_{\alpha, n+i} \neq 0$ and $y_{\alpha, i}=0$, then $y_{\alpha, j}=0$ for $j=0, \ldots, m$. Hence we have $\pi\left(V_{\alpha, n+i}\right) \subset W_{\alpha, i}$, from which we infer readily that

$$
\pi^{-1}\left(W_{\alpha, i}\right)=V_{\alpha, n+i} \cup \bigcup_{j=0}^{n-1} V_{\alpha, j} \cap \pi^{-1}\left(W_{\alpha, i}\right)
$$

If we check the system of coordinate transformations of the line bundles $\mathcal{O}_{P(E \oplus F \oplus 1)}(1)$ and $\mathcal{O}_{P\left(\bar{q}^{*} E \oplus L\right)}(1)$ with respect to the refined open covering $\left\{V_{\alpha, n+i}, V_{\alpha, j} \cap \pi^{-1}\left(W_{\alpha, i}\right)\right\}$, then we have $\varpi^{*} \mathcal{O}_{P(E \oplus F \oplus 1)}(1)=\mathcal{O}_{P\left(\bar{q}^{*} E \oplus L\right)}(1)$.

Now we prove the theorem. Let $\varpi_{X}: \tilde{P}_{X} \rightarrow P\left(T^{*} X \oplus f^{*} T^{*} Y \oplus 1\right)\left(\varpi_{Y}\right.$ : $\tilde{P}_{Y} \rightarrow P\left(T^{*} X \oplus f^{*} T^{*} Y \oplus 1\right)$, resp. $)$ be the blow-up of $P\left(T^{*} X \oplus f^{*} T^{*} Y \oplus 1\right)$ along $P\left(f^{*} T^{*} Y\right)\left(P\left(T^{*} X\right)\right.$, resp. $)$ and $\pi_{X}: \tilde{P}_{X} \rightarrow P\left(T^{*} X \oplus 1\right)\left(\pi_{Y}: \tilde{P}_{Y} \rightarrow P\left(f^{*} T^{*} Y \oplus\right.\right.$ 1), resp.) the morphism induced by the projection.

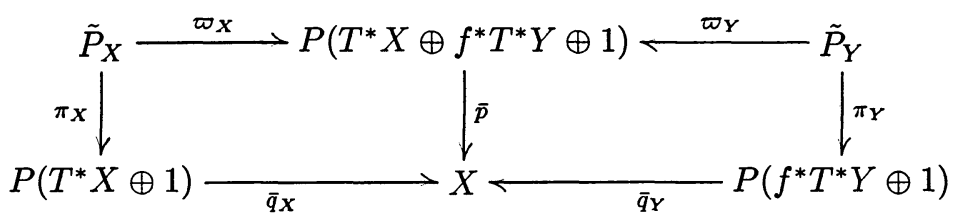

Then by Lemma $\pi_{X}: \tilde{P}_{X} \rightarrow P\left(T^{*} X \oplus 1\right)\left(\pi_{Y}: \tilde{P}_{Y} \rightarrow P\left(f^{*} T^{*} Y \oplus 1\right)\right.$, resp. $)$ is considered to be the projective bundle $P\left(\bar{q}_{X}{ }^{*} f^{*} T^{*} Y \oplus L_{X}\right) \rightarrow P\left(T^{*} X \oplus 1\right)\left(P\left(\bar{q}_{Y}{ }^{*} T^{*} X \oplus\right.\right.$ $\left.L_{Y}\right) \rightarrow P\left(f^{*} T^{*} Y \oplus 1\right)$, resp.), where $L_{X}\left(L_{Y}\right.$, resp.) is the tautological line bundle on $P\left(T^{*} X \oplus 1\right)\left(P\left(f^{*} T^{*} Y \oplus 1\right)\right.$, resp.). The vector bundle $T^{*} X \oplus f^{*} T^{*} Y$ is an open dense subset of $P\left(T^{*} X \oplus f^{*} T^{*} Y \oplus 1\right)$ and the subvarieties $P\left(f^{*} T^{*} Y\right), P\left(T^{*} X\right)$ are contained in the complement of $T^{*} X \oplus f^{*} T^{*} Y$. Hence we may consider $T^{*} X \oplus f^{*} T^{*} Y=$ $T^{*} X \times_{X} f^{*} T^{*} Y$ to be an open dense subset of $\tilde{P}_{X}$ and $\tilde{P}_{Y}$, respectively. It is clear that $\varpi_{X *} \pi_{X}^{*}\left(\left[\overline{T_{Z}^{*} X}\right]\right)=\left[\overline{T_{Z}^{*} X \times \times_{X} f^{*} T^{*} Y}\right]$ on $P\left(T^{*} X \oplus f^{*} T^{*} Y \oplus 1\right)$. Hence by Fulton[2, Example 3.2.1.] we have

$$
\left.\bar{q}_{X *} \pi_{X *}\left(s\left(\tilde{L}_{X}\right) \cap \pi_{X}{ }^{*}\left[\overline{T_{Z}^{*} X}\right]\right)=\bar{q}_{X *}\left(s\left(\bar{q}_{X}{ }^{*} f^{*} T^{*} Y \oplus L_{X}\right)\right) \cap\left[\overline{T_{Z}^{*} X}\right]\right) .
$$

Hence we have

$$
\begin{gathered}
s\left(f^{*} T^{*} Y\right) \cap s^{*}(Z, X)=\bar{q}_{X *} \pi_{X *}\left(s\left(\tilde{L}_{X}\right) \cap \pi_{X}{ }^{*}\left[\overline{T_{Z}^{*} X}\right]\right) \\
=\bar{p}_{*} \varpi_{X *}\left(s\left(\tilde{L}_{X}\right) \cap \pi_{X}^{*}\left[\overline{T_{Z}^{*} X}\right]\right)=\bar{p}_{*}\left(s(L) \cap \varpi_{X *} \pi_{X}{ }^{*}\left[\overline{T_{Z}^{*} X}\right]\right) \\
=\bar{p}_{*}\left(s(L) \cap\left[\overline{T_{Z}^{*} X \times_{X} f^{*} T^{*} Y}\right]\right)
\end{gathered}
$$

in the Borel-Moore homology group $H .(Z, \mathbb{Z})$.

Meanwhile we show that the section $\tilde{f}^{*} \alpha_{Y}: f^{*} T^{*} Y \rightarrow T^{*} X \oplus f^{*} T^{*} Y=q_{Y}{ }^{*} T^{*} X$, where $q_{Y}: f^{*} T^{*} Y \rightarrow X$ is the projection, is extensible to the section $\sigma$ to the vector bundle $L_{Y}^{-1} \otimes \bar{q}_{Y}{ }^{*} T^{*} X$ over $P\left(f^{*} T^{*} Y \oplus 1\right)$. It is sufficient to see this locally. Hence we assume that $X$ (Y, resp.) is an open subset of $\mathbb{C}^{n}\left(\mathbb{C}^{m}\right.$, resp.) with coordinates $\left(x_{i}\right)\left(\left(y_{j}\right)\right.$, resp. $)$ and the morphism $f$ is given by $\left(y_{j}\right)=f\left(\left(x_{i}\right)\right)=\left(y_{j}\left(x_{i}\right)\right)$. Let 
$\left(\xi_{i}\right)\left(\left(\eta_{j}\right)\right.$, resp. $)$ the associated fiber coordinates of $T^{*} X\left(T^{*} Y\right.$, resp.). Then $P\left(f^{*} T^{*} Y \oplus\right.$ $1)=X \times \mathbb{P}\left(\mathbb{C}^{m} \times \mathbb{C}\right)$, where $\mathbb{P}\left(\mathbb{C}^{m} \times \mathbb{C}\right)$ is a projective space with the homogeneous coordinates $\left(\eta_{0}, \ldots, \eta_{m-1}, \eta_{m}\right)$. Let $V_{j}$ be the open subset of $\mathbb{P}\left(\mathbb{C}^{m} \times \mathbb{C}\right)$ such that $\eta_{j} \neq 0$. We consider $\pi_{Y}{ }^{-1}\left(X \times V_{j}\right)$ to be $X \times V_{j} \times \mathbb{P}\left(\mathbb{C}^{n} \times \mathbb{C}\right)$, where $\mathbb{P}\left(\mathbb{C}^{n} \times \mathbb{C}\right)$ is a projective space with homogeneous coordinate $\left(\left(\xi_{i}\right), \zeta_{j}\right)$. Then $T^{*} X \oplus f^{*} T^{*} Y$ is the open subset of $\pi_{Y}^{-1}\left(X \times V_{j}\right)$ such that $\zeta_{j} \neq 0$. The 1 -form $\tilde{f}^{*} \alpha_{Y}$ is expressed as

$$
\tilde{f}^{*} \alpha_{Y}=\sum \eta_{j} \frac{\partial y_{j}}{\partial x_{i}} d x_{i} .
$$

Hence the section $\tilde{f}^{*} \alpha_{Y}$ is defined by the equations

$$
\xi_{i}=\sum_{j=0}^{m-1} \frac{\partial y_{j}}{\partial x_{i}} \eta_{j}, \quad i=0, \ldots, n-1 .
$$

We define $\sigma \mid V_{j}: X \times V_{j} \rightarrow X \times V_{j} \times \mathbb{P}\left(\mathbb{C}^{n} \times \mathbb{C}\right)$ to be

$$
\left(\sigma \mid V_{j}\right)\left(\left(x_{i}\right),\left(\eta_{0}, \ldots, \eta_{m}\right)\right)=\left(\left(x_{i}\right),\left(\eta_{0}, \ldots, \eta_{m}\right),\left(\left(\sum_{\nu=0}^{\nu=m-1} \frac{\partial y_{\nu}}{\partial x_{i}} \eta_{\nu}\right), \eta_{j}\right)\right) .
$$

These $\left\{\sigma \mid V_{j}\right\}$ give the desired extension.

By the above defining equations of the section we see easily that the image of the section $\sigma$ is the cone over the image of the restriction of $\sigma$ to $P\left(f^{*} T^{*} Y\right)$.

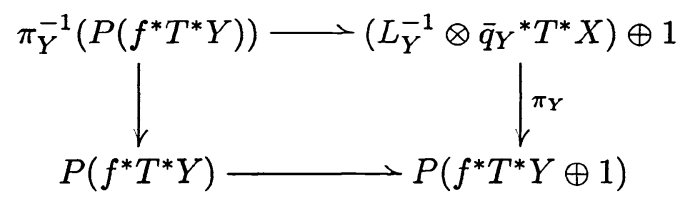

Hereafter we often use the smame letter for the fundamental cycle of a subvariety. It is clear that the closure $\overline{p_{X}^{*} T_{Z}^{*} X}$ of $p_{X}^{*} T_{Z}^{*} X$ in $\tilde{P}_{Y}$ is a cone over a subvariety of $\pi_{Y}^{-1}\left(P\left(f^{*} T^{*} Y\right)\right)$, where $p_{X}$ is the projecton of $q_{X}^{*}\left(T^{*} X\right)$ to $T^{*} X$, for the defining equations of the subvariety $p_{X}^{*} T_{Z}^{*} X$ in $\tilde{P}_{Y}$ do not contain $\left(\eta_{i}\right)$ of the above coordinate systems. Therefore the intersection of $p_{X}^{*} T_{Z}^{*} X$ and the image of the section $\sigma$ is the cone over the intersection of the corresponding subvarieties in $\pi_{Y}^{-1}\left(P\left(f^{*} T^{*} Y\right)\right)$. Hence we have

$$
\overline{p_{X}^{*}\left(T_{Z}^{*} X\right) \cdot \tilde{f}^{*}\left(\alpha_{Y}\right)\left(\left[f^{*} T^{*} Y\right]\right)}=\overline{p_{X}^{*} T_{Z}^{*} X} \cdot \sigma\left(P\left(f^{*} T^{*} Y \oplus 1\right)\right) .
$$

By Fulton[2, Proposition 3.3.] we have

$$
\overline{p_{X}^{*} T_{Z}^{*} X} \cdot \sigma\left(P\left(f^{*} T^{*} Y \oplus 1\right)\right)=\pi_{*}\left(c_{n}(\zeta) \cap \overline{p_{X}^{*} T_{Z}^{*} X}\right),
$$

where $\zeta$ is the universal rank $n$ quotient bundle of $\left(L_{Y}^{-1} \otimes \bar{q}_{Y}^{*} T^{*} X\right) \oplus 1$. Since $\varpi_{Y *}(\Theta \cdot C)=0$ for any cycle $C$ on $\tilde{P}_{Y}$, we have

$$
\varpi_{Y *}\left(c_{n}(\zeta) \cap \overline{p_{X}^{*} T_{Z}^{*} X}\right)=\varpi_{Y *}\left(c_{n}\left(\left(L_{Y}^{-1} \otimes \bar{q}_{Y}^{*} T^{*} X\right) \oplus 1\right) \cap \overline{p_{X}^{*} T_{Z}^{*} X}\right),
$$

for, letting $\tilde{L}_{Y}$ be the tautological line bundle on the projective bundle $P\left(\left(L_{Y}^{-1} \otimes\right.\right.$ $\left.\left.\bar{q}_{Y}{ }^{*} T^{*} X\right) \oplus 1\right)$, we have the exact sequence

$$
0 \rightarrow \tilde{L}_{Y} \rightarrow \pi^{*}\left(\left(L_{Y}^{-1} \otimes \bar{q}_{Y}^{*}\left(T^{*} X\right) \oplus 1\right) \rightarrow \zeta \rightarrow 0,\right.
$$


and by Lemma we have $c_{1}\left(\tilde{L}_{Y}^{-1}\right)=\Theta$. From $\tilde{L}_{Y}^{-1}=\mathcal{O}_{P\left(\bar{q}_{Y}{ }^{*} T^{*} X \oplus L_{Y}\right)}(1) \otimes L_{Y}$ we have

$$
\varpi_{Y *}\left(c_{1}\left(L_{Y}^{-1}\right)^{i} \cap C\right)=\varpi_{Y *}\left(c_{1}\left(\mathcal{O}_{P\left(\bar{q}_{Y}{ }^{*} T^{*} X \oplus L_{Y}\right)}(1)\right)^{i} \cap C\right)
$$

for a positive intager $i$ and a cycle $C$ on $\tilde{P}_{Y}$ and hence we have

$$
\begin{gathered}
\varpi_{Y *}\left(c_{n}\left(\left(L_{Y}^{-1} \otimes \bar{q}_{Y}^{*} T^{*} X\right) \oplus 1\right) \cap \overline{p_{X}^{*} T_{Z}^{*} X}\right) \\
=\varpi_{Y *}\left(\sum_{i=0}^{n} c_{1}\left(\mathcal{O}_{P\left(\bar{q}_{Y^{*}} T^{*} X \oplus L_{Y}\right)}(1)\right)^{i} c_{n-i}\left(\bar{q}^{*} T^{*} X\right) \cap \overline{p_{X}^{*} T_{Z}^{*} X}\right) \\
=\sum_{i=0}^{n} c_{1}\left(\mathcal{O}_{P\left(T^{*} X \oplus f^{*} T^{*} Y \oplus 1\right)}(1)\right)^{i} \bar{p}^{*} c_{n-i}\left(T^{*} X\right) \cap \overline{T_{Z}^{*} X \times_{X} f^{*} T^{*} Y} .
\end{gathered}
$$

Therefore we have

$$
\begin{gathered}
\left.\bar{q}_{Y *}\left(s\left(L_{Y}\right) \cap \overline{\left[p_{X}^{*}\left(T_{Z}^{*} X\right) \cdot \tilde{f}^{*}\left(\alpha_{Y}\right)\left(\left[f^{*} T^{*} Y\right]\right)\right.}\right]\right) \\
=\bar{p}^{*}\left(\sum_{k \geq 0,0 \leq i \leq n} c_{1}\left(\mathcal{O}_{P\left(\bar{q}_{Y}{ }^{*} T^{*} X \oplus L_{Y}\right)}(1)\right)^{k+i} \bar{p}^{*} c_{n-i}\left(T^{*} X\right) \cap \overline{T_{Z}^{*} X \times_{X} f^{*} T^{*} Y}\right) .
\end{gathered}
$$

By the assumption that $n \leq m$, noting that

$$
\left.\pi_{X *}\left(c_{1}\left(\mathcal{O}_{P\left(f^{*} T^{*} Y \oplus 1\right)}(1)\right)^{i} \cap \pi_{X}^{*}(C)\right)\right)=0
$$

for $i \leq m$ and a cycle on $P\left(T^{*} X \oplus 1\right)$, we have

$$
\begin{gathered}
\pi_{X *}\left(\sum_{i} \sum_{k \geq 0} c_{1}\left(\mathcal{O}_{P\left(\bar{q}_{Y}^{*} T^{*} X \oplus L_{Y}\right)}(1)\right)^{k+i} \pi_{X}^{*}\left(c_{n-i}\left(\bar{q}_{X}^{*} T^{*} X\right) \cap T_{Z}^{*} X\right)\right. \\
=\pi_{X *}\left(\sum_{i} \sum_{k \geq 0} c_{1}\left(\mathcal{O}_{P\left(\bar{q}_{Y}^{*} T^{*} X \oplus L_{Y}\right)}(1)\right)^{k} \pi_{X}^{*}\left(c_{n-i}\left(\bar{q}_{X}^{*} T^{*} X\right) \cap T_{Z}^{*} X\right)\right. \\
=\pi_{X *}\left(s\left(\tilde{L}_{X}\right) \cap \pi_{X}^{*}\left(\bar{q}_{X}^{*} c\left(T^{*} X\right) \cap T_{Z}^{*} X\right)\right)=s\left(f^{*} T^{*} Y \oplus L_{X}\right) \bar{q}_{X}^{*} c\left(T^{*} X\right) \cap T_{Z}^{*} X .
\end{gathered}
$$

Thus we have

$$
\left.\bar{q}_{Y *}\left(s\left(L_{Y}\right) \cap \overline{\left[p_{X}^{*}\left(T_{Z}^{*} X\right) \cdot \tilde{f}^{*}\left(\alpha_{Y}\right)\left(\left[f^{*} T^{*} Y\right]\right)\right.}\right]\right)=s\left(f^{*} T^{*} Y\right) c\left(T^{*} X\right) \cap s^{*}(Z, X),
$$

which completes the proof of the theorem.

Remark If, for example, $m=0$, we have the following formula

$$
\check{c}_{M, 0}(Z)=\bar{q}_{X *}\left(s_{0}(X) \cdot\left[T_{Z}^{\bar{*}} X\right]\right),
$$

where $s_{0}$ is the zero section to the projective completion $P\left(T^{*} X \oplus 1\right)$. 
Finally we prove the corollary. In a similar notations to the above we have the following fiber square

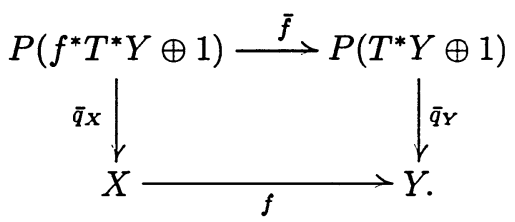

Let $L_{Y}$ be the tautological line bundle on $P\left(T^{*} Y \oplus 1\right)$. Then the induced bundle $\bar{f}^{*} L_{Y}$ is the tautological line bundle on $P\left(f^{*} T^{*} Y \oplus 1\right)$. Hence by Theorem 2 we have

$$
\begin{aligned}
& f_{*}\left(\check{c}_{M}\left(\mathcal{M}^{*}\right)\right)=f_{*}\left(\sum m_{j} c\left(T^{*} X\right) \cap s^{*}\left(\bar{V}_{j}, X\right)\right) \\
& \quad=f_{*}\left(\sum m_{j} c\left(f^{*} T^{*} Y\right) \cap \bar{q}_{X *}\left(s\left(\bar{f}^{*} L_{Y}\right) \cap \overline{\left[p_{X}^{*}\left(T_{V_{j}}^{*} X\right) \cdot \tilde{f}^{*}\left(\alpha_{Y}\right)\left(\left[f^{*} T^{*} Y\right]\right)\right.}\right]\right) \\
& \quad=c\left(T^{*} Y\right) \cap \bar{q}_{Y *} \bar{f}_{*}\left(\sum m_{j} s\left(\bar{f}^{*} L_{Y}\right) \cap \overline{\left[p_{X}^{*}\left(T_{\bar{V}_{j}}^{*} X\right) \cdot \tilde{f}^{*}\left(\alpha_{Y}\right)\left(\left[f^{*} T^{*} Y\right]\right)\right]}\right) .
\end{aligned}
$$

By Theorem 1 we have

$$
\bar{f}_{*}\left(\sum m_{j} \overline{\left[p_{X}^{*}\left(T_{\bar{V}_{j}}^{*} X\right) \cdot \tilde{f}^{*}\left(\alpha_{Y}\right)\left(\left[f^{*} T^{*} Y\right]\right)\right]}\right)=\overline{\operatorname{Ch}\left(\int_{f} \mathcal{M}^{*}\right)}
$$

Hence we have

$$
\begin{gathered}
\left.f_{*}\left(\check{c}_{M}\right)\left(\mathcal{M}^{*}\right)=c\left(T^{*} Y\right) \cap \bar{q}_{Y *}\left(s\left(L_{Y}\right) \cap \overline{\left[\operatorname{Ch}\left(\int_{f} \mathcal{M}^{\cdot}\right)\right.}\right]\right) \\
=c\left(T^{*} Y\right) \cap s^{*}\left(\int_{f} \mathcal{M}, Y\right)=\check{c}_{M}\left(\int_{f} \mathcal{M}^{*}\right) .
\end{gathered}
$$

\section{REFERENCES}

[1] J. L. BRYLinski, A. DUbSon, And M. KashiwarA, Formule de l'indice pour les modules holonomes et obstruction d'Euler locale, C. R. Acad. Sci., 293 (1981), pp. 573-576.

[2] W. Fulton, Intersection Theory, Springer-Verlag, 1984.

[3] V. Ginsburg, Characteristic cycles and vanishing cycles, Invent. Math., 84 (1986), pp. 327402.

[4] M. Kashiwara And P. Schapira, Sheaves on Manifolds, Springer-Verlag, 1990.

[5] S. KAWAI, A note on the characteristic cycle of the image of the constant sheaf, Comm. Math. Univ. Snacti Pauli, 48 (1999), pp. 119-128.

[6] R. MacPherson, Chern class for singular varieties, Ann. Math., 100 (1974), pp. 423-432.

[7] C. SABBAH, Quelques remarques sur la géométrie des espaces conormaux, in Systèmes différentiels et singularités, Astérisque 130, 1985, pp. 161-192. 
